\title{
GROUP RINGS WITH SIMPLE AUGMENTATION IDEALS
}

\author{
K. BONVAllet, B. HARTLEY, D. S. PASSMAN ${ }^{1}$ AND M. K. SMITH
}

\begin{abstract}
ABSTRACr. Group algebras of algebraically closed groups and of universal groups are shown to have simple augmentation ideals and to be primitive.
\end{abstract}

In recent years a large number of examples of primitive group rings have been constructed. In this note we offer some additional examples. However here primitivity is really a secondary consideration since it follows from the even more surprising property that in these group rings $K[G]$ the augmentation ideal $\omega(K[G])$ is the unique proper ideal. The following theorem has a rather unwieldly hypothesis. Nevertheless, as will be apparent, it is precisely what is needed to handle the families of algebraically closed groups and universal groups.

THEOREM. Let $G$ be a simple group, let $K$ be a field and let $q$ be a prime different from the characteristic of $K$. Suppose that for any finite number of distinct elements $1=x_{0}, x_{1}, \ldots, x_{n} \in G$ there exist elements $y_{0}, y_{1}, \ldots, y_{n}$ $\in G$ such that

(1) $\left\langle y_{i}^{x_{j}} \mid i=0,1, \ldots, n, j=0,1, \ldots, n\right\rangle$ is an elementary abelian q-group.

(2) $\left\langle\left(y_{i}, x_{i}\right) \mid i=1,2, \ldots, n\right\rangle$ has order precisely $q^{n}$.

(3) $\left\langle y_{0}^{x_{j}} \mid j=0,1, \ldots, n\right\rangle$ has order precisely $q^{n+1}$.

Then $\omega(K[G])$ is the unique proper ideal of $K[G]$. Furthermore $K[G]$ is primitive.

Proof. Suppose that $I$ is a nonzero proper ideal of $K[G]$. We proceed in a series of steps.

Step 1. Let $\alpha \in I, \alpha \neq 0$. Then we can assume that 1 occurs in the support of $\alpha$ and we write $\alpha=\sum_{0}^{n} k_{i} x_{i}^{-1}$ with $1=x_{0}, x_{1}, \ldots, x_{n}$ distinct elements of $G$ and with $k_{0} \neq 0$. We apply the hypothesis of this theorem to these elements and let $y_{1}, y_{2}, \ldots, y_{n}$ be given as in (1) and (2). Thus by (1) if $A$ is the group $A=\left\langle y_{i}^{x_{j}} \mid i=1,2, \ldots, n, j=0,1, \ldots, n\right\rangle$ then $A$ is an elementary abelian $q$ group. Set $z_{i}=\left(y_{i}, x_{i}\right)=y_{i}^{-1} x_{i}^{-1} y_{i} x_{i}$ for $i=1,2, \ldots, n$.

We show now by inverse induction on $s$ with $n \geqslant s \geqslant 0$ that $I$ contains an element

$$
\beta_{s}=\sum_{i=0}^{s} \beta_{s i} x_{i}^{-1}
$$

Received by the editors March 11, 1975.

AMS (MOS) subject classifications (1970). Primary 16A26, 20C05; Secondary 16A20, 20F25.

Key words and phrases. Group ring, augmentation ideal, primitive ring, algebraically closed group, universal group, wreath product.

1 Passman's research supported in part by NSF Grant GP-32813X.

C American Mathematical Society 1976 
with $\beta_{s i} \in K[A]$ and such that for $s<n$

$$
\beta_{s 0}=k_{0}\left(z_{n}-1\right)\left(z_{n-1}-1\right) \cdots\left(z_{s+1}-1\right) .
$$

First for $x=n$ we merely take $\beta_{s}=\alpha$. Now suppose we have $\beta_{s}$ as above contained in $I$ with $s>0$. Then $y_{s}^{-1} \beta_{s} y_{s}$ and $z_{s} \beta_{s}$ both belong to $I$ and hence

$$
\beta_{s-1}=z_{s} \beta_{s}-y_{s}^{-1} \beta_{s} y_{s} \in I .
$$

Furthermore since $A$ is abelian and $y_{s}, z_{s} \in A$ we have

$$
\begin{aligned}
\beta_{s-1} & =\sum_{i=0}^{s} z_{s} \beta_{s i} x_{i}^{-1}-\sum_{i=0}^{s} y_{s}^{-1} \beta_{s i} x_{i}^{-1} y_{s} \\
& =\sum_{i=0}^{s} \beta_{s i}\left\{z_{s}-\left(y_{s}, s_{i}\right)\right\} x_{i}^{-1} \\
& =\sum_{i=0}^{s-1} \beta_{s i}\left\{z_{s}-\left(y_{s}, x_{i}\right)\right\} x_{i}^{-1}
\end{aligned}
$$

since $z_{s}=\left(y_{s}, x_{s}\right)$. Thus since $x_{0}=1, \beta_{s-1,0}$ has the appropriate form and the induction step is proved.

In particular, when $s=0$ we conclude that

$$
\beta_{0}=k_{0}\left(z_{n}-1\right)\left(z_{n-1}-1\right) \cdots\left(z_{1}-1\right) \in I .
$$

Furthermore $k_{0} \neq 0$ and

$$
\left\langle z_{1}, z_{2}, \ldots, z_{n}\right\rangle=\left\langle z_{1}\right\rangle \times\left\langle z_{2}\right\rangle \times \cdots \times\left\langle z_{n}\right\rangle
$$

is a direct product of cyclic groups of order $q$ by property (2). Hence $\beta_{0} \neq 0$ and we have shown that there exists a finite elementary abelian $q$-subgroup $A$ of $G$ with $I \cap K[A] \neq 0$.

Step 2. Let $A$ be a finite elementary abelian $q$-subgroup of $G$, whose existence is guaranteed by Step 1 , such that $I \cap K[A] \neq 0$. Write $A$ $=\left\{1=x_{0}, x_{1}, \ldots, x_{n}\right\}$ and let $y_{0} \in G$ be given satisfying (1) and (3). Thus by (1), if $B$ is the group $B=\left\langle y_{0}^{x_{j}} \mid j=0,1, \ldots, n\right\rangle$, then $B$ is an elementary abelian $q$-group normalized by $A$. Furthermore since $|B|=q^{n+1}$ and $n+1$ $=|A|$, it is clear that $A$ acts faithfully on $B$ so $A \cap B=\langle 1\rangle$. Thus we conclude first that $H=B A$ is the semidirect product of $B$ by $A$ and then that $H \simeq Z_{q} \sim A$ with $Z_{q}$ corresponding to the cyclic group $\left\langle y_{0}\right\rangle$. It follows that $3(H)$, the center of $H$, is the cyclic group of order $q$ generated by $z$ $=y_{0}^{x_{0}} y_{0}^{x_{1}} \cdots y_{0}^{x_{n}}$.

Let $i$ be fixed and let $B_{i}=\left\{\left(b, x_{i}\right) \mid b \in B\right\}$. Since $B$ is abelian the map $B \rightarrow B_{i}$ given by $b \rightarrow\left(b, x_{i}\right)$ is easily seen to be a homomorphism onto. Hence $B_{i}$ is a subgroup of $B$ and $B_{i} \simeq B / C_{B}\left(x_{i}\right)$ since $C_{B}\left(x_{i}\right)$ is clearly the kernel of the homomorphism. It then follows easily in $K[G]$ that

$$
\sum_{b \in B}\left(x_{i}^{-1}\right)^{b}=\sum_{b \in B}\left(b, x_{i}\right) x_{i}^{-1}=\left|\mathbf{C}_{B}\left(x_{i}\right)\right| \hat{B}_{i} x_{i}^{-1}
$$

where $\hat{B}_{i}$ denotes the sum of the elements of $B_{i}$.

Now let $\alpha=\sum k_{i} x_{i}^{-1} \in I \cap K[A]$ with $k_{0} \neq 0$. Then $\beta=\sum_{b \in B} b^{-1} \alpha b$ 
$E I$ and by the above

$$
\beta=\sum_{i} k_{i}\left|\mathbf{C}_{B}\left(x_{i}\right)\right| \hat{B}_{i} x_{i}^{-1} .
$$

For $i=0$, since $x_{0}=1$ the summand here is $k_{0}|B| x_{0}^{-1}=k_{0}|B|$. On the other hand if $i \neq 0$ then $x_{i}$ does not centralize $B$ so $B_{i}$ is a nonidentity subgroup of $B$. Furthermore since both $A$ and $B$ are abelian, we conclude easily that $B_{i} \triangle H$ and hence since $H$ is a $q$-group this yields $B_{i} \cap 3(H) \neq\langle 1\rangle$. Thus $z \in B_{i}$ so $(z-1) \hat{B}_{i}=0$ and we have

$$
(z-1) \beta=k_{0}|B|(z-1) \text {. }
$$

Finally $(z-1) \beta \in I, k_{0} \neq 0$ and $|B| \neq 0$ in $K$ since by assumption $q$ $\neq \operatorname{char} K$. We have therefore shown that there exists a nonidentity element $z \in G$ with $z-1 \in I$.

Step 3. Let $H=\{h \in G \mid h-1 \in I\}$. Then since $I$ is an ideal it follows that $H$ is a normal subgroup of $G$. Furthermore by Step 2 we have $H \neq\langle 1\rangle$. Hence since $G$ is simple we conclude that $H=G$ and this implies immediately that $I \supseteq \omega(K[G])$. But $\omega(K[G])$ is a maximal ideal of $K[G]$ so this yields $I=\omega(K[G])$ and we have therefore obtained our main assertion.

Finally apply the hypothesis of the theorem with $1=x_{0}$. Then by (3), $G$ has a cyclic subgroup $C$ of order $q$ and hence $e=1-\hat{C} / q$ is a nonzero idempotent of $K[G]$. Furthermore, nonzero idempotents are never contained in the Jacobson radical of a ring so there exists an irreducible $K[G]$-module $V$ with $V e \neq 0$. Since $e \in \omega(K[G])$ this yields $V \omega(K[G]) \neq 0$ and thus the zero ideal is the only possibility for the kernel of the action of $K[G]$ on $V$. This means that $V$ is a faithful irreducible $K[G]$-module so $K[G]$ is primitive and the Theorem is proved.

To see where the above elements $y_{i}$ might come from, we make the following simple observation.

Lemma. Let $1=x_{0}, x_{1}, \ldots, x_{n}$ be distinct elements of $G$ and let $A$ $=\left\langle y_{0}, y_{1}, \ldots, y_{n}\right\rangle$ be an elementary abelian $q$-group of order $q^{n+1}$. If $A$ and $G$ are suitably embedded in the wreath product $A \sim G$, then the elements $x_{i}$ and $y_{i}$ satisfy conditions (1), (2) and (3) of the Theorem.

We now use this to handle some interesting families of groups. A group $G$ is said to be algebraically closed [3] if every finite system $W_{i}\left(x_{j}, y_{k}\right)=1$ and $\bar{W}_{i}\left(x_{j}, y_{k}\right) \neq 1$ of word equations and word inequalities, in the variables $y_{k}$ and group elements $x_{j}$, which has a simultaneous solution in some group extension of $G$ also has a solution in $G$. Such groups are quite plentiful and in fact, by [3, Theorem 1], every infinite group can be embedded in an algebraically closed group of the same cardinality.

COROLlary 1. Let $G$ be an algebraically closed group and let $K$ be a field. Then $\omega(K[G])$ is the unique proper ideal of $K[G]$ and the group ring is primitive.

Proof. By [2], $G$ is a simple group. Fix a prime $q$ different from the characteristic of $K$ and let $1=x_{0}, x_{1}, \ldots, x_{n}$ be finitely many distinct elements of $G$. Then by the Lemma there exists a group extension of $G$ having elements $y_{0}, y_{1}, \ldots, y_{n}$ satisfying conditions (1), (2) and (3) of the Theorem. 
But observe that condition (1) is merely a finite set of commuting and order equations and that, given (1), conditions (2) and (3) amount to a finite set of inequalities. Thus since $G$ is algebraically closed these equations and inequalities must also have a solution in $G$. Thus the Theorem applies and the result follows.

Other groups of interest are the universal groups of $\mathrm{Ph}$. Hall. A group $G$ is universal (see [1, Chapter 6]) if it is locally finite, contains copies of all finite groups and has the property that any two isomorphic finite subgroups are conjugate. Such groups are reasonably numerous and indeed, by [1, Theorem 6.5 , every infinite locally finite group can be embedded in a universal group of the same cardinality.

Corollary 2. Let $G$ be a universal group and let $K$ be a field. Then $\omega(K[G])$ is the unique proper ideal of $K[G]$ and the group ring is primitive.

Proof. By [1, Theorem 6.1(d)] $G$ is simple. Fix a prime $q$ different from the characteristic of $K$ and let $1=x_{0}, x_{1}, \ldots, x_{n}$ be finitely many distinct elements of $G$. Then $H=\left\langle x_{0}, x_{1}, \ldots, x_{n}\right\rangle$ is a finite group, since $G$ is locally finite. By the Lemma, if $A=\left\langle y_{0}, y_{1}, \ldots, y_{n}\right\rangle$ is an elementary abelian $q$-group of order $q^{n+1}$ then the elements $x_{i}$ and $y_{i}$ in $A \sim H$ satisfy properties (1), (2) and (3) of the Theorem. But by [1, Theorem 6.1(b)] the embedding of $H$ into $G$ can be extended to an embedding of $A \sim H$ into $G$. Therefore $G$ satisfies the hypothesis of the Theorem and the result follows.

Finally let $G$ be an arbitrary group. If $H$ is a proper normal subgroup of $G$, then $I=\omega(K[H]) \cdot K[G]$ is a proper ideal of $K[G]$ distinct from the augmentation ideal. Thus a necessary condition for $\omega(K[G])$ to be the unique proper ideal of the group ring is that $G$ be simple. On the other hand this condition is by no means sufficient. Consider for example $G=\mathrm{Alt}_{\Omega}$ where $\Omega$ is an infinite set and each element of $G$ moves only finitely many points. Of course $G$ is simple. Let $K$ by any field and form the permutation module $V$ for $K[G]$. That is, $V$ has as a $K$-basis the elements of $\Omega$ and $G$ acts on $V$ by appropriately permuting this basis. If $\sigma$ and $\tau$ are two disjoint permutations in $G$, for example take $\sigma=(123)$ and $\tau=(456)$, then it is easy to see that $(\sigma-1)(\tau-1) \neq 0$ acts trivially on $V$ but that $\sigma-1$ does not. Hence the kernel of the action of $K[G]$ on $V$ is a proper ideal different from $\omega(K[G])$.

\section{REFERENCES}

1. O. H. Kegel and B. A. F. Wehrfritz, Locally finite groups, North-Holland, Amsterdam, 1973.

2. B. H. Neumann, A note on algebraically closed groups, J. London Math. Soc. 27 (1952), 247-249. MR 13, 721.

3. W. R. Scott, Algebraically closed groups, Proc. Amer. Math. Soc. 2 (1951), 118-121. MR 12, 671.

Department of Mathematics, University of Wisconsin, Madison, Wisconsin 53706 (Current address of D. S. Passman)

Department of Mathematics, University of Warwick, Coventry, Warwickshire, CV4 7AL, ENGLAND (Current address of B. Hartley)

Department of Mathematics, University of Texas, Austin, Texas 78712 (Current address of M. K. Smith)

Current address (K. Bonvallet): The Prudential Insurance Company of America, Newark, New Jersey 07102 